The authors have previously identified a single nucleotide polymorphism 13,910 base pairs upstream of the LPH gene, which is associated with the persistence/nonpersistence of lactase during maturation. The $\mathrm{C} / \mathrm{T}_{-13910}$ and $\mathrm{T} / \mathrm{T}_{-13910}$ genotypes are associated with lactase persistence during maturation, whereas the $\mathrm{C} / \mathrm{C}_{-13910}$ variant is consistent with nonpersistence and adult-type hypolactasia.

The new study included 329 children of African, Finnish and 'other white' backgrounds, who underwent gastrointestinal endoscopy for abdominal problems. DNA from intestinal biopsy specimens was sequenced in order to determine the frequencies of the three genotypes. The samples were also assayed for lactase, maltase and sucrase activities.

The C/C genotype was the predominant variant in the African and 'other white' patients, but was recorded in only $14.7 \%$ of the Finnish participants. These frequencies correlate with the prevalence of adult-type hypolactasia in these populations. In children over 12 years old, the $\mathrm{C} / \mathrm{C}$ genotype predicted low lactase activity with a sensitivity of $93.3 \%$ and a specificity of $100 \%$.

The authors conclude that this genetic test is a useful method of diagnosing adult-type hypolactasia, especially since it can be performed using only a drop of peripheral blood and can be semi-automated.

Original article Rasinperä $\mathrm{H}$ et al. (2004) A genetic test which can be used to diagnose adult-type hypolactasia in children. Gut 53: 1571-1576

\section{Familial pancreatic adenocarcinoma: risk factors and clinical characteristics}

Around $5-10 \%$ of pancreatic adenocarcinoma patients have a familial predisposition to the disease. Little is known about the clinical characteristics of this familial form, however, and it has yet to be properly defined. A recent US study by James and colleagues has made use of registry data to compare the familial and sporadic forms of the disease.

Of 826 patients diagnosed with pancreatic adenocarcinoma between 1972 and 1999, $30(3.6 \%)$ had at least one parent, sibling or child with the disease (the 'familial' cohort), whereas the remainder had no family history (the 'sporadic' cohort). Both groups had similarly advanced stage at presentation, poor overall survival and low resectability rates. There was no significant difference between the average age at diagnosis in the two groups, but patients in the familial cohort were significantly more likely to present at age $<50$ years than those with sporadic disease $(36 \%$ vs $18 \%, P=0.017)$. There was a higher proportion of smokers in the familial cohort than in the sporadic group ( $87 \%$ vs $66 \%$ ) of those with documented history of smoking habits; although this difference was not statistically significant $(P=0.06)$, smoking may be an environmental trigger for familial disease and so is relevant in terms of risk assessment.

James et al. note that the younger age at onset of familial pancreatic carcinoma should be considered when developing screening strategies for at-risk patients. They also recommend that patients with a family history of the disease should be strongly advised to avoid smoking.

Original article James TA et al. (2004) Risk factors associated with earlier age of onset in familial pancreatic carcinoma. Cancer 101: 2722-2726

\section{Capsule endoscopy for esophageal imaging}

A new 'PillCam' ingestible capsule might be a feasible alternative to conventional upper endoscopy for assessing patients with suspected esophageal disorders. A pilot study by Eliakim et al. has compared the two methods in 17 patients with heartburn and/or epigastric pain, showing that the PillCam is safe, accurate, well-tolerated and convenient. Importantly, this so-called 'capsule endoscopy' approach is performed without sedation and so might be superior in terms of safety.

The esophageal PillCam Diagnostic System, developed by Given Imaging Ltd, was modified from a similar device designed for small bowel imaging. The patient swallows the $3-\mathrm{cm}$-long capsule in a supine position and is raised to $60^{\circ}$ over a period of 4 minutes. During its transit through the esophagus, the disposable capsule collects video images from each end. These are transmitted digitally to a data recorder unit via sensor arrays and then analyzed with proprietary 